\title{
FENÓMENOS RELACIONADOS CON EL USO DE METÁFORAS EN EL DISCURSO DEL PROFESOR. EL CASO DE LAS GRÁFICAS DE FUNCIONES
}

\author{
Font Moll, Vicenç y Acevedo Nanclares, Jorge Iván \\ Departamento de Didáctica de las CCEE y de la Matemática \\ Universitat de Barcelona
}

\begin{abstract}
Resumen. En este artículo comentamos un fenómeno que se observa al analizar el discurso del profesor cuando explica la representación gráfica de funciones en el bachillerato: el profesor usa expresiones que sugieren, entre otras, metáforas del tipo «la gráfica de una función se puede considerar como la traza que deja un punto que se mueve sobre la gráfica». En la primera parte presentamos el marco teórico sobre la interdependencia entre cuerpo y mente propuesto por Lakoff y Núñez. En la segunda comentamos las diferentes metáforas que han estructurado el concepto de gráfica de una función. En la tercera se describe el estudio de un profesor -siguiendo la secuencia: $a$ ) grabación en vídeo de la clase; $b$ ) entrevista posterior; y $c$ ) análisis de las producciones de los alumnos- que muestra que el profesor usa de manera poco consciente estas metáforas y cree que sus efectos en la comprensión de sus alumnos son inocuos. Finalizamos con unas conclusiones en las que se analizan las posibles causas de este fenómeno.
\end{abstract}

Palabras clave. Didáctica, matemáticas, análisis, gráficas, metáfora.

Summary. In this paper, we comment a phenomenon that is observed when analyzing the teacher's discourse when explaining of the graphical representation of functions at high school: the teacher uses expressions that suggest, among others, metaphors like «The graph of a function can be considered as the trace of a point that moves over the graph». In the first part, we present the theoretical framework about the embodiment of mind proposed by Lakoff and Núñez. In the second part, we comment the different metaphors that have structured the concept of graphic of a function. In the third part we describe the study of a teacher-following the sequence: $a$ ) video-recording of a class, $b$ ) interviews, $c$ ) analysis of the productions of the students- which shows that the teacher uses these metaphors in a not completely conscious way and that he believes that their effects in the understanding of the students are innocuous. We end up with some conclusions about the possible causes of this phenomenon.

Keywords. Didactics, mathematics, analysis, graphs, metaphor.

\section{INTRODUCCIÓN}

Las definiciones de los contenidos matemáticos que aparecen en los libros de texto son productos que esconden el proceso histórico que las ha generado. Por ello, para comunicar estos contenidos en el proceso de enseñanzaaprendizaje, el profesorado de bachillerato ${ }^{1}$ no se limita únicamente a dar su definición, sino que se vale también de diferentes recursos para facilitar la comprensión a sus alumnos. Entre los diferentes recursos que se suelen utilizar, destaca de forma preferente el uso de metáforas.

La investigación que presentamos trata sobre los fenómenos relacionados con el uso de metáforas por parte del profesor al explicar la representación gráfica de funciones en el bachillerato. En este trabajo hemos puesto de manifiesto que se produce, con una cierta generalidad, el siguiente fenómeno: el profesor usa de manera poco consciente expresiones que sugieren, entre otras, metáforas del tipo: «La gráfica de una función se puede considerar como la traza que deja un punto que se mueve sujeto a determinadas condiciones.» $\mathrm{O}$ bien una variación de esta metáfora: «La gráfica de una función se puede considerar como la traza que deja un punto que se mueve sobre la gráfica.» Dichas metáforas no son inocuas y producen efectos significativos en la comprensión de los alumnos. 


\section{PENSAMIENTO METAFÓRICO}

Desde que Lakoff y Johnson (1991) pusieron de manifiesto la importancia del pensamiento metafórico, entendido como la interpretación de un campo de experiencias en términos de otro ya conocido, el papel del pensamiento metafórico en la formación de los conceptos matemáticos es un tema que cada vez tiene más relevancia para la investigación en didáctica de las matemáticas (English, 1997; Lakoff y Núñez, 1998, 2000; Núñez, 2000; Núñez y Lakoff, 1998; Pimm, 1990; Van Dormolen, 1991).

Las metáforas se caracterizan por crear, entre un dominio de partida y un dominio de llegada, un puente conceptual que permite la transfusión de propiedades del dominio de partida en el domino de llegada. En otras palabras, crean un cierto «isomorfismo» que permite que se transpongan una serie de características y estructuras. Ahora bien, las metáforas sólo dejan ver un aspecto del dominio de llegada que no engloba su totalidad. La metáfora nos sirve para mostrar el aspecto que deseamos evidenciar y oculta otros aspectos, de los cuales muchas veces ni siquiera somos conscientes.

Las investigaciones sobre el pensamiento metafórico han detectado diferentes clases de metáforas. Un primer grupo lo constituyen las de tipo extramatemático, como la de «una función es una máquina», que sirven para explicar o interpretar situaciones matemáticas en términos de situaciones reales. Uno de los ejemplos más notables de este tipo es la del «contenedor», usada para estructurar la teoría de clases, la cual, según Núñez (2000), es una metáfora inconsciente, que tiene sus raíces en la vida cotidiana y que podemos visualizar de la siguiente manera (Núñez, 2000, p. 13):
Un segundo grupo, frecuente en las aulas, lo constituyen las metáforas extramatemáticas, las cuales permiten estructurar partes del conocimiento matemático a partir de otras partes ya conocidas. Ejemplos de este tipo son: «los números reales son los puntos de una recta», «los números complejos son vectores», «las funciones de proporcionalidad son rectas que pasan por el origen de coordenadas», etc.

Esta última metáfora ilustra cómo esta figura lingüística sirve para conectar diferentes sentidos y, por tanto, amplía el significado que tiene para una persona un determinado objeto matemático. En efecto, supongamos que un alumno ha estudiado el concepto de pendiente de una recta en un contexto geométrico, de manera que entiende que la pendiente de una recta «es la longitud del segmento BC de la figura». Es decir, el alumno entiende que el segmento BC determina la inclinación de la recta al determinar el ángulo que forma la recta con el eje horizontal.

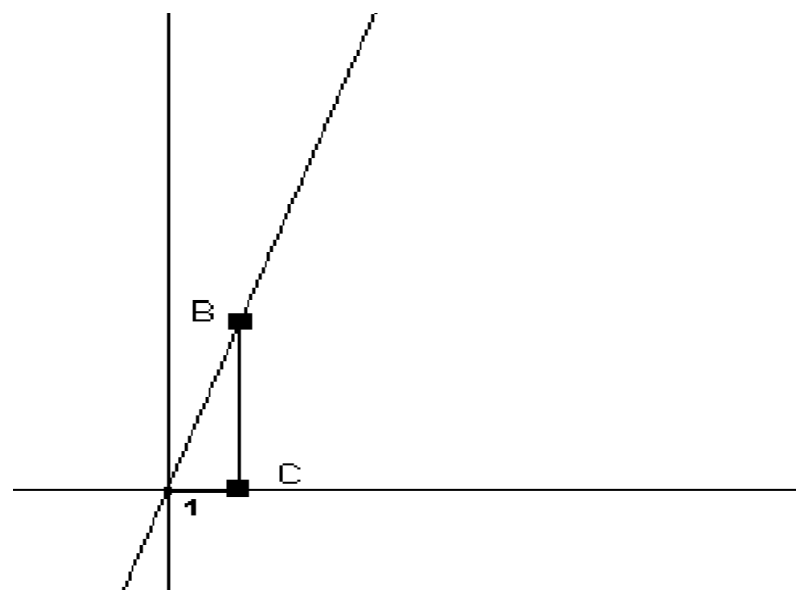

Las clases son contenedores

\begin{tabular}{|l|l|l|}
\hline \multicolumn{1}{|c|}{$\begin{array}{c}\text { Dominio de partida } \\
\text { Esquema del contenedor }\end{array}$} & \multicolumn{1}{c|}{$\begin{array}{c}\text { Domino de llegada } \\
\text { Clases }\end{array}$} \\
\hline Interior del contenedor & $\longrightarrow$ & Clase \\
\hline Objetos dentro del contenedor & $\longrightarrow$ & Miembros de la clase \\
\hline Ser un objeto del interior & $\longrightarrow$ & La relación de pertenencia \\
\hline Un interior de un contenedor dentro de uno más grande & $\longrightarrow$ & Una subclase de la clase más grande \\
\hline Superponer el interior de dos contenedores & $\longrightarrow$ & Intersección de dos clases \\
\hline La totalidad de los interiores de dos contenedores & $\longrightarrow$ & La unión de clases \\
\hline El exterior de un contenedor & $\longrightarrow$ & El complementario de la clase \\
\hline
\end{tabular}


Si posteriormente el mismo alumno trata la función de proporcionalidad en un contexto algebraico/funcional con actividades ${ }^{2}$ como la siguiente, variará su forma de entender la pendiente de una recta:

Actividad: El entrenador de un corredor de fondo le está cronometrando el tiempo. En los primeros 16 segundos los resultados son:

\begin{tabular}{|c|c|c|c|c|c|c|}
\hline Tiempo (s) & 0 & 2 & 4 & 6 & 8 & 16 \\
\hline Espacio (m) & 0 & 6 & 12 & 18 & 24 & $\ldots$ \\
\hline
\end{tabular}

a) ¿Qué se puede decir de la marcha del corredor? ¿A qué velocidad ha ido durante estos 16 segundos?

b) Busca la fórmula de la función que nos da el espacio recorrido (en $\mathrm{m}$ ) en función del tiempo (en s).

c) Dibuja la gráfica de esta función.

d) ¿Cuál es la ordenada que corresponde a la abscisa $x=1$ ? ¿Crees que esta ordenada determina el ángulo que forma la recta con el eje de abscisas?

e) ¿Qué relación hay entre la pendiente y el número de la fórmula de la función que multiplica a la $x$ ?

En este contexto algebraico/funcional el concepto de pendiente toma dos sentidos que el alumno puede relacionar entre sí: «la pendiente de una función de proporcionalidad es el número que multiplica a la $x$ en la fórmula $y=a x » y$ «la pendiente de una función de proporcionalidad es el número que corresponde a $1 »$. De esta manera el alumno ha estudiado el concepto de pendiente en dos contextos diferentes: el geométrico y el algebraico/funcional y con tres sentidos diferentes. Con la metáfora «la función de proporcionalidad es una recta que pasa por el origen de coordenadas» se consigue relacionar estos tres sentidos entre sí y también con la palabra pendiente.

Sin embargo, el uso de metáforas plantea algunas dificultades. Puede ocurrir, por ejemplo, que el alumno, en lugar de entender que una función se puede comprender a partir del funcionamiento de una máquina, tome la expresión «una función es una máquina» de manera literal y que piense que una función es realmente una máquina. Dicho con otras palabras: la expresión anterior tiene un significado literal, pero también tiene un segundo significado, que es el que queremos que entienda el alumno. Queremos que el alumno estructure su conocimiento sobre funciones a partir de su conocimiento sobre las máquinas, no que piense que una función es una máquina. Para que comprenda que no debe tomar la expresión «una función es una máquina» de manera literal, se ha de producir un conflicto cognitivo entre el significado literal de la expresión y el contexto en el que se usa. La existencia de este conflicto es lo que hace que el alumno busque un significado diferente del literal. Esta situación se produce con una cierta frecuencia, ya que el profesor a menudo usa metáforas de manera incontrolada sin ser consciente de que lo hace. Ahora bien, las dificultades relacionadas con el pensamiento metafórico van más allá de la dificultad causada por un conflicto con el significado literal, ya que, incluso, cuando el uso que se hace de la metáfora es correcto, se corre el riesgo de trasladar relaciones que no son válidas en el dominio de llegada.

\section{UNA NUEVA TEORÍA COGNITIVA DE LA MATE- MÁTICA}

La importancia que tiene el pensamiento metafórico en la construcción del significado de los objetos matemáticos es reconocida por una gran mayoría de los investigadores en didáctica de las matemáticas y es el origen de una nueva teoría sobre qué son las matemáticas propuesta por Lakoff y Núñez (2000). La nueva disciplina, llamada por sus autores «ciencia cognitiva de la matemática» (Lakoff y Núñez, 2000; Núñez, 2000), tiene por objetivo estudiar, de manera empírica y multidisciplinar, las ideas matemáticas de las personas como una materia científica. El núcleo central de esta nueva teoría está basado en la importancia que tiene el cuerpo sobre la mente y en los relativamente recientes hallazgos en lingüística cognitiva. Su tesis afirma que el origen de las estructuras matemáticas que construyen las personas, y también las que se construyen en instituciones, hay que buscarlo en los procesos cognoscitivos cotidianos, como son los esquemas de las imágenes y el pensamiento metafórico. Según estos autores, dichos procesos permiten explicar cómo la construcción de los objetos matemáticos, tanto los personales como los institucionales, está sostenida por la manera cómo nuestro cuerpo se relaciona con los objetos de la vida cotidiana.

Según este punto de vista, la naturaleza de las matemáticas hay que buscarla en las ideas de las personas, no en las demostraciones formales, axiomas y definiciones ni tampoco en mundos trascendentes platónicos. Dichas ideas surgen de los mecanismos cognitivos y corporales de las personas. Debido a su origen, común a todas las personas, las ideas matemáticas no son arbitrarias, no son el producto de convenciones completamente sociales y culturales, aunque las dimensiones sociohistóricas jueguen papeles importantes en la formación y desarrollo de estas ideas. En la misma línea que la teoría de Piaget (1979), esta nueva teoría afirma que las matemáticas son el resultado de la experiencia humana, pero no es el resultado de puras convenciones sociales, ya que, por razones de tipo evolutivo, todos desarrollamos los mismos mecanismos cognitivos, y de ellos surgen las ideas matemáticas.

Recientemente, la ciencia cognitiva no dualista en la que se basa la propuesta de Lakoff y Núñez ha realizado importantes avances en la comprensión del funcionamiento de la mente y más en concreto en lo que atañe a nuestra comprensión de las matemáticas. Son los siguientes: 1) La importancia que tiene el cuerpo sobre la mente. La naturaleza y dinámica de nuestros cuerpos, nuestros cerebros y nuestro 
funcionamiento de todos los días tienen una importancia fundamental en la estructura de la razón humana, la cual incluye el pensamiento matemático. 2) El papel del conocimiento inconsciente. La mayoría de los procesos cognitivos son inconscientes, en el sentido de que no son accesibles a nuestra introspección consciente, puesto que no podemos llegar directamente a nuestros sistemas conceptuales y a nuestros procesos cognitivos de nivel inferior que incluyen una gran parte del pensamiento matemático. 3) El pensamiento metafórico. La mayor parte de los seres humanos conceptúan conceptos abstractos en términos concretos y usan la estructura inferencial y maneras de razonar conectadas con nuestro sistema motórico y sensorial. El mecanismo cognitivo que permite que lo abstracto se comprenda en términos de lo concreto es la metáfora conceptual. El pensamiento matemático también hace uso de ella cuando conceptúa números como puntos en una línea, o el espacio como conjunto de puntos.

Este punto de vista considera que la actual investigación en neuropsicología ha demostrado que todos los individuos de la especie homo sapiens nacen con la capacidad de distinguir entre un número muy pequeño de objetos y sucesos -como en el caso de la subitización, proceso que nos permite ver que un conjunto tiene 3 o 4 elementos sin necesidad de contarlos- y con la de efectuar cálculos aritméticos muy simples con números muy pequeños. Estos hallazgos son importantes para la comprensión de los rudimentos biológicos de aritmética básica. Sin embargo, ellos nos dicen muy poco sobre la complejidad de las matemáticas, ya que éstas van mucho más allá de la aritmética y requieren un aparato cognitivo enorme, muy superior al que desarrollan los bebés y animales o del que desarrolla un adulto sin una instrucción específica. A la pregunta «¿Cuáles son las capacidades cognitivas, basadas en la importancia del cuerpo sobre la mente, que permiten a una persona pasar de las habilidades numéricas básicas innatas a un entender profundo y rico propio de las matemáticas de una licenciatura universitaria de una facultad de ciencias?», Lakoff y Núñez responden que éstas no son independientes del aparato cognitivo usado fuera de la matemática. Según estos autores, la estructura cognitiva necesaria para la matemática avanzada se vale del mismo aparato conceptual que el pensamiento cotidiano en las situaciones ordinarias no matemáticas, esto es: esquemas de la imagen, esquemas aspectuales, mezclas conceptuales y metáfora conceptual. De todos estos procesos, es el pensamiento metafórico el más importante para la construcción de las matemáticas.

Este punto de vista, según nuestra opinión, es en cierta forma apriorístico, ya que considera que la actividad constitutiva del sujeto en el acto de comprensión matemática lleva a verdades consideradas necesarias para cualquier sujeto normal. Por una parte, considera probado por la actual neuropsicología que todas las personas nacen con la capacidad de distinguir entre un número muy pequeño de objetos y sucesos; $y$, por otra parte, considera que casi todos los sujetos tienen la capacidad de llegar a comprender las verdades matemáticas, puesto que se basan en unos procesos cognitivos básicos y comunes a todos los miembros de la especie. Ahora bien, en nuestra opinión, se trata de un tipo de apriorismo que resulta relativamente débil si lo comparamos con otras teorías sobre qué son las matemáticas.

\section{METÁFORAS RELACIONADAS CON LAS GRÁ- FICAS DE FUNCIONES}

Según Font (2000), las gráficas se han estructurado históricamente a partir de las siguientes metáforas: $a$ ) las curvas son secciones; $b$ ) las curvas son la traza que deja un punto que se mueve sujeto a determinadas condiciones; $c$ ) las curvas son la traza que deja un punto que se mueve sujeto a determinadas condiciones el análisis de las cuales permite encontrar una ecuación que cumplen los puntos de la curva. Hasta principios del siglo XIX, cuando Cauchy empieza la reorganización del análisis infinitesimal, esta última metáfora es la que se puede encontrar en los libros de análisis infinitesimal. Es decir, hasta la aritmetización del análisis, las gráficas de funciones eran consideradas como la trayectoria descrita por un punto en movimiento, la cual se podía expresar por una fórmula. Esta manera de entender las gráficas de funciones queda muy clara en la obra de Newton, donde podemos hallar constantes referencias a un punto que se mueve sobre una parábola, una hipérbola, etc. A partir de los trabajos, entre otros, de Fourier, Cauchy y Dirichlet, se aceptaron, como gráficas de funciones, curvas que no podían ser trayectorias. Con la posterior aplicación de la teoría de conjuntos a las funciones, terminó de coger cuerpo la metáfora conjuntista: $d$ ) la gráfica de una función $f$ es el conjunto formado por los puntos de coordenadas $(x, f(x))^{3}$.

En este artículo nos limitaremos a analizar los efectos que produce la metáfora «La gráfica de una función se puede considerar como la traza que deja un punto que se mueve sujeto a determinadas condiciones»-o bien una variación de esta metáfora: «La gráfica de una función se puede considerar como la traza que deja un punto que se mueve sobre la gráfica» en la enseñanza de la representación gráfica de funciones en el bachillerato.

En la investigación explicada en Font $(2000)^{4}$ se describe una situación de enseñanza-aprendizaje en la que alumnos de bachillerato utilizan las siguientes construcciones, realizadas con el programa Cabri, con el objetivo de ayudarles a entender que la recta tangente es la recta a la cual se aproximan las rectas secantes:

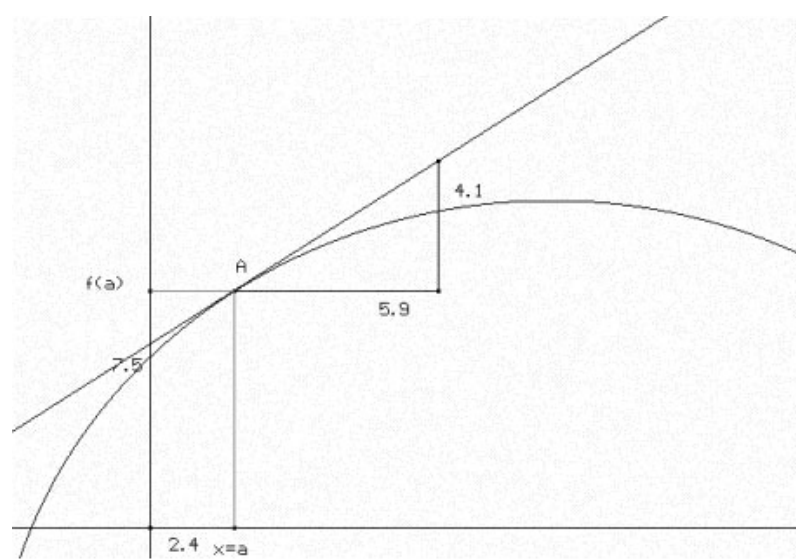




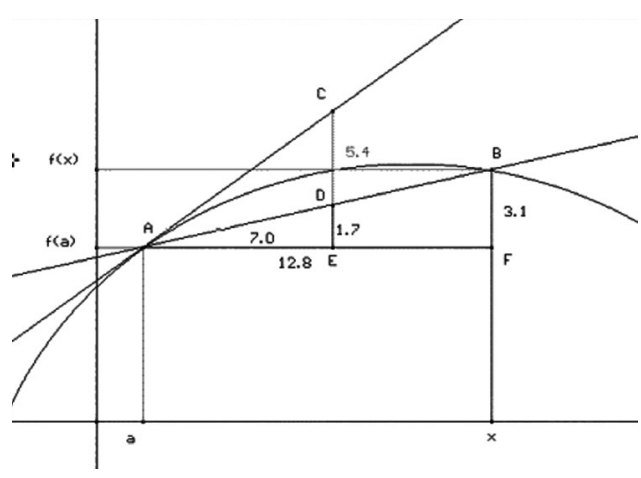

Uno de los fenómenos observados, cuando los alumnos experimentaban con estas construcciones, fue que el hecho de utilizar la metáfora «La gráfica de una función se puede considerar como la traza que deja un punto que se mueve sobre la gráfica» de manera inconsciente en el discurso del profesor causó la siguiente dificultad en algunos alumnos: «[...] observamos que había alumnos que, cuando movían el punto A, pensaban que el nuevo punto continuaba siendo el punto A y que la nueva recta tangente era la misma que antes pero con diferente inclinación. De hecho, es como si estructurasen la situación en términos de una persona que se mueve (punto A) con un saco en la espalda (recta tangente) por una carretera que primero sube y después baja (gráfica) y considerasen que la persona y el saco siempre son los mismos, a pesar de estar en diferentes lugares y tener diferente inclinación.» (Font, 2000, p. 122).

La investigación citada anteriormente tenía como objetivo principal analizar los significados institucionales y personales implicados en un proceso de estudio del objeto derivada en una institución escolar de bachillerato. En dicha investigación se observó el fenómeno anterior pero éste sólo se comenta de manera secundaria y se propone su estudio como una posible línea de desarrollo. El hecho de que este fenómeno fuese de suficiente entidad para ser el objetivo de un estudio específico nos llevó a diseñar una nueva investigación para contrastarlo y para ampliarlo.

\section{OBJETIVOS Y CARACTERÍSTICAS DE LA IN- VESTIGACIÓN}

El objetivo de esta investigación era comprobar que este fenómeno detectado en Font (2000) ocurre con una cierta regularidad. Concretamente nos planteamos los siguientes objetivos:

1) Detectar si el profesor, al explicar la representación gráfica de funciones en el bachillerato, usa metáforas del tipo «La gráfica de una función se puede considerar como la traza que deja un punto que se mueve sujeto a determinadas condiciones» -o bien una variación de esta metáfora: «La gráfica de una función se puede considerar como la traza que deja un punto que se mueve sobre la gráfica.»

2) Analizar el contenido de su discurso para detectar, además de la anterior, otras metáforas.
3) Verificar si el profesor es consciente del uso que ha hecho de las metáforas en su discurso y hasta qué punto las tiene controladas.

4) Determinar el efecto que producen estas metáforas sobre los alumnos.

Las características metodológicas de la investigación fueron de tipo interpretativo. Por una parte fue: $a$ ) etnográfica: ya que se pretendió comprender los acontecimientos tal y como los interpretaban los sujetos investigados, mediante una inmersión en su pensamiento y en su práctica; $b$ ) puntual: la información fue obtenida en un período de tiempo relativamente breve; $c$ ) de campo: la información se obtuvo en el lugar de trabajo de los sujetos investigados.

Los instrumentos utilizados fueron: $a$ ) la observación de las clases impartidas por diferentes profesores; $b$ ) la grabación en vídeo de una clase de un profesor; c) a partir del análisis de esta grabación se diseñó un cuestionario para realizar una entrevista personalizada y semiestructurada con este profesor y un cuestionario que tuvieron que responder 9 de sus alumnos.

Los profesores que participaron en esta investigación lo hicieron de manera voluntaria y consintieron de forma consciente la intromisión en sus tareas docentes (observación de sus clases, grabación en vídeo, análisis de materiales de trabajo...). Los alumnos participaron a petición de su profesor. La elección del profesor grabado en vídeo y la de 9 de sus alumnos no fue realizada bajo ningún criterio estadístico.

\section{EL USO DE METÁFORAS EN EL DISCURSO EXPLICATIVO DEL PROFESOR. EL CASO DE LAS GRÁFICAS}

Después de analizar el discurso de diferentes profesores al impartir el tema de la representación gráfica de funciones en el bachillerato, comprobamos el uso generalizado de la metáfora «La gráfica de una función se puede considerar como la traza que deja un punto que se mueve sujeto a determinadas condiciones» -o bien una variación de esta metáfora: «La gráfica de una función se puede considerar como la traza que deja un punto que se mueve sobre la gráfica». Este hecho nos llevó a considerar la necesidad de realizar el estudio de un caso particular, con el objetivo de analizar el uso de diferentes tipos de metáforas en su discurso. Para tal fin se escogió un profesor al azar. El profesor escogido imparte clases de bachillerato en el IES Vilassar de Mar, de la provincia de Barcelona.

\section{Grabación en vídeo de una clase}

La grabación en vídeo que se comenta en esta investigación corresponde a la clase impartida por este profesor el 21 de marzo de 2001 en una clase de $1^{\circ}$ de bachillerato (16 años) mientras explicaba el tema de la representación gráfica de funciones. La filmación dura 45 minutos, de los cuales, en este artículo, por cuestiones de espacio, se comentan ${ }^{5}$ tan sólo dos fragmentos no consecutivos de 8 minutos en total. 


\begin{tabular}{|c|c|}
\hline Texto del vídeo & Observaciones \\
\hline . & . \\
\hline PRIMER FRAGMENTO & \\
\hline ........me parece que está todo & $\begin{array}{l}\text { Después de haber hecho todo el estudio analítico de la función } y=2 x /\left(x^{2}-1\right) \text { (dominio, puntos } \\
\text { de corte con los ejes, crecimiento, y decrecimientos, máximos y mínimos, concavidad y } \\
\text { convexidad, puntos de inflexión y asíntotas), el profesor se dispone a dibujar la gráfica. }\end{array}$ \\
\hline
\end{tabular}

......Pues ahora vamos a ver el esbozo de la función bien hecho ....ahora....pues, sí que tenemos a "-1" una asíntota....eh!....

A ver!.. fijaos..... hemos dicho.....bueno ya veíamos como.....venga.

¡La asíntota vertical eh!.. a la derecha de la asíntota vertical, es coger de más infinito........infinito y pasa por cero cero que tenemos un punto de inflexión que es donde hay un cambio y da una cosa así.

\section{Y luego aquí viene así}

Y aquí viene así
El profesor empieza dibujando los ejes de coordenadas y ubica en los dos ejes los signos $+\infty$ y $-\infty$. A continuación traza las asíntotas verticales que cortan el eje de abscisas en $1 \mathrm{y}-1$.

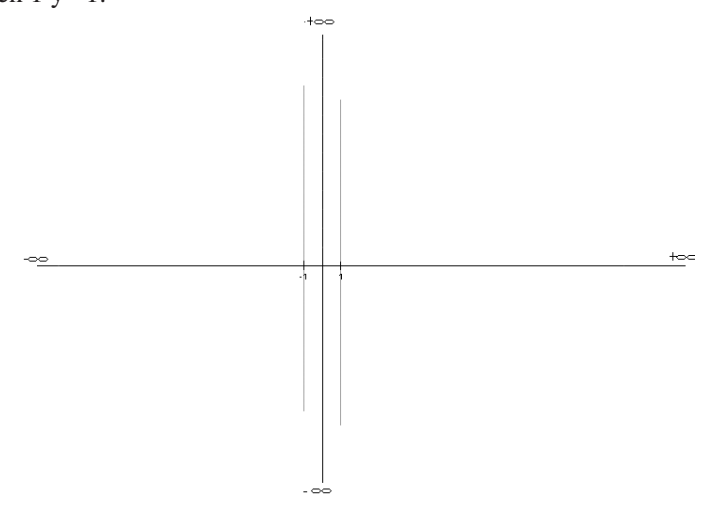

El profesor empieza a trazar la parte central de la gráfica de la función. Empieza por más infinito y termina en menos infinito.

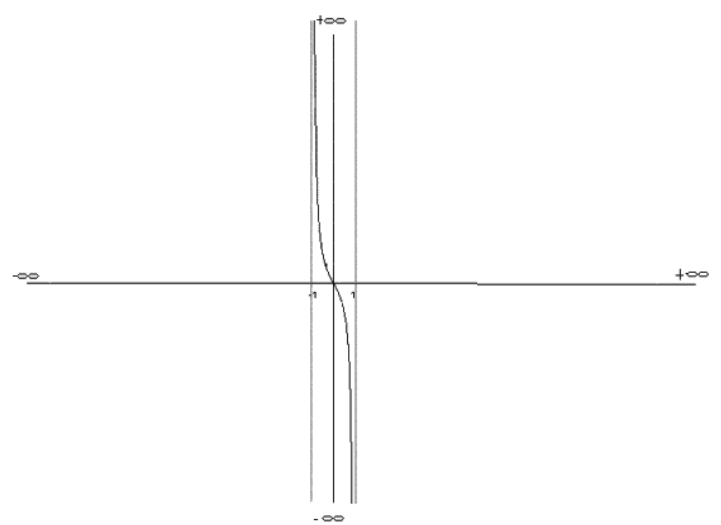

Hace el trazado de la parte izquierda de la gráfica.

Hace el trazado de la parte derecha de la gráfica y mueve las manos para reforzar sus comentarios sobre la gráfica.

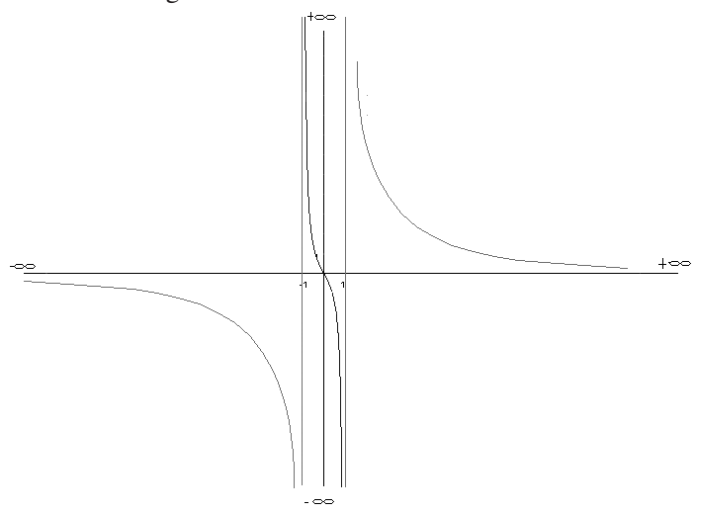


¡Eh! ...

Comparad la coherencia...... de este esbozo con todo lo que hemos dicho antes, eh!...

Fijaos que a veces...vamos, con lo dicho hasta ahora... ...eh!...hacéis el proceso bien y después hacéis el esbozo una cosa rara.

Señala la gráfica.

Interpretad bien el significado de estos resultados, ha de ser una cosa de este estilo.

Bueno, hemos hecho esta función racional que no tiene..........hemos hecho esta función racional que no tiene racio........ pero tiene dos asíntotas verticales, con la dificultad que representa hacer el análisis de la función en sus laterales, y una asíntota horizontal con $y=0$.

El otro día hicimos, ¿cuáles hemos hecho?

Hace esta pregunta pero no da tiempo a que los alumnos la respondan.

Hemos hecho.

Sigue explicando cosas de clases anteriores y recordando algunas gráficas trabajadas anteriormente en clase.

\begin{tabular}{|c|c|}
\hline Texto del vídeo & Observaciones \\
\hline (n.............................. & (n) \\
\hline SEGUNDO FRAGMENTO & \\
\hline Quería proponer una que...jeh!.. la viéseis.. que sería esta....... & Escribe la función $y=x /\left(x^{2}+1\right)$. \\
\hline $\begin{array}{l}\text { Esta función racional que presenta otra característica,.. aquí por } \\
\text { ejemplo el dominio......... }\end{array}$ & \\
\hline 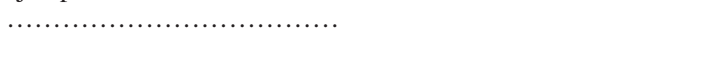 & $\begin{array}{l}\text { Continúa hablando de otra función cuya gráfica se había representado } \\
\text { anteriormente. }\end{array}$ \\
\hline $\begin{array}{l}\text { También podemos hacer ésta, que es relativamente sencilla, pero } \\
\text { tiene su interés...... } \\
\text { Todavía me quedan cinco minutos de clase } \ldots \ldots \ldots \ldots \ldots \text {....... vamos a } \\
\text { ver......................... }\end{array}$ & $\begin{array}{l}\text { Los alumnos ya quieren terminar la clase, pero el profesor insiste en } \\
\text { que vean esa función. } \\
\text { Los alumnos continúan hablando. }\end{array}$ \\
\hline $\begin{array}{l}\text { Vamos a empezar un poco ésta, porque tiene algo nuevo que no } \\
\text { ha aparecido hasta hoy. }\end{array}$ & \\
\hline $\begin{array}{l}\text { Hasta ahora todas las funciones racionales que hemos hecho, } \\
\text { hemos encontrado que el dominio no eran todos los reales. } \\
\text { ¡Vale!.... }\end{array}$ & \\
\hline 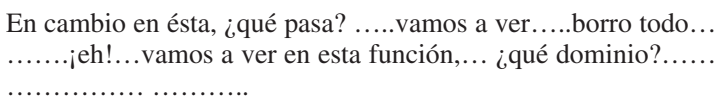 & El profesor empieza el estudio analítico de la función $y=x /\left(x^{2}+1\right)$ \\
\hline $\begin{array}{l}\text { Lo primero que.... lo primero entonces que hacíamos era } \\
\text { el dominio eh!.. y el dominio como lo buscábamos....que } \\
\text { decíamos!..... decíamos... existirá para cualquier valor que esta } \\
\text { función de } x \text { excepto para aquéllos que anulen el denominador, } \\
\text { y para comprobarlo, pues igualábamos el denominador a } \\
\text { cero.................... }\end{array}$ & $\begin{array}{l}\text { Escribe en la pizarra, igualando el denominador a cero y despejando la } \\
x \text {, obteniendo raíces cuadradas de números negativos. }\end{array}$ \\
\hline $\begin{array}{l}\text { Por lo tanto, esta función existe siempre, el dominio será todo } R \\
\mathrm{y} \text {, por lo tanto, no tendrá ninguna asíntota vertical...... }\end{array}$ & \\
\hline
\end{tabular}




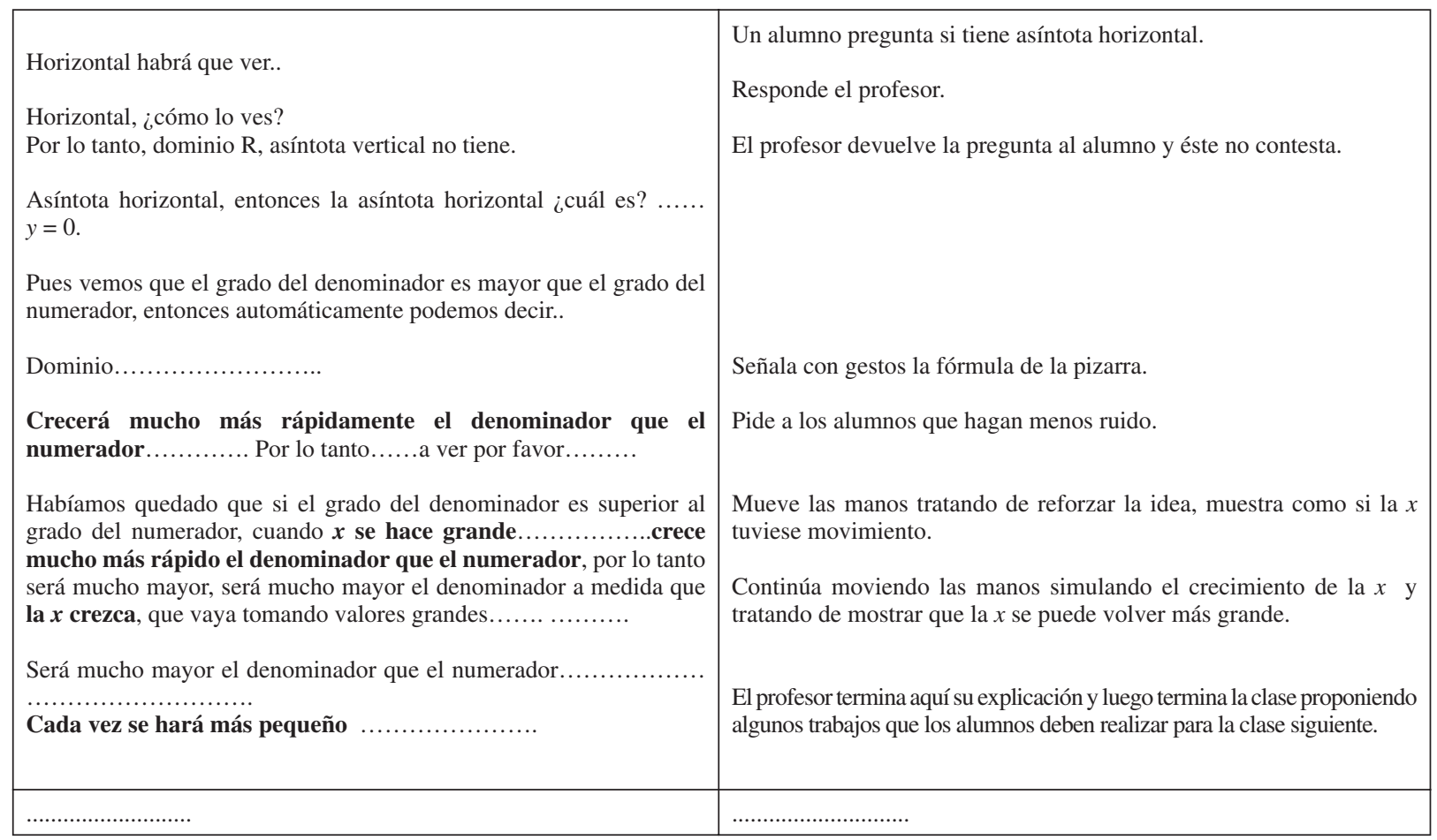

De la anterior trascripción podemos observar, entre otros, los siguientes hechos significativos:

1) El profesor ha hecho uso de diferentes metáforas en su discurso.

2) Podemos ver, en primera instancia, cómo ha utilizado un discurso dinámico, acompañado de gestos con sus manos, para explicar los componentes estáticos de la gráfica. En este discurso, la metáfora «La gráfica de una función se puede considerar como la traza que deja un punto que se mueve sujeto a determinadas condiciones»-o bien una variación de esta metáfora, «La gráfica de una función se puede considerar como la traza que deja un punto que se mueve sobre la gráfica»-, está presente en frases como «viene por aquí», «pasa por cero cero», etc.

3) Por otra parte, ha supuesto que los números sean objetos que crecen y que pueden aumentar de tamaño, al usar frases como: «el denominador crece más rápido», « $x$ se hace grande», «la $x$ crece», etc. Estas metáforas, al igual que la comentada en 2, tienen su dominio fuera del campo de las matemáticas. En la terminología de Lakoff y Núñez (2000) se pueden considerar como grounding metaphora.
4) El crecimiento y decrecimiento de la gráfica se explica en términos visual-dinámicos con frases como: «por aquí decrece porque viene así».

Una vez evidenciado que las metáforas son un recurso cotidiano en el discurso del profesor, de acuerdo con nuestro tercer objetivo, nos propusimos investigar en qué medida su uso está favoreciendo o dificultando el aprendizaje de los alumnos. Un primer paso en esta línea es conocer si el profesor hace uso de las metáforas de una manera controlada y si es consciente de los efectos que produce su uso en el aprendizaje de los alumnos. A tal efecto, entrevistamos al profesor cuya clase había sido grabada en vídeo.

\section{Entrevista}

La siguiente entrevista se realizó el pasado 5 de abril de 2001, cuando eran las 11 de la mañana en un aula del instituto Vilassar del Mar, en la localidad que lleva el mismo nombre. La entrevista duró 30 minutos, de los cuales se transcriben tan sólo algunos fragmentos. 


\begin{tabular}{|c|c|}
\hline Texto & Observaciones \\
\hline $\begin{array}{l}E \text {. En el vídeo que he visto dices frases como: Aquí viene así, viene por aquí, } \\
\text { pasa por cero cero, } x \text { crece, va por aquí, crece más el numerador que el de- } \\
\text { nominador. Y cuando lo haces, indicas movimientos con tus manos. ¿Qué } \\
\text { quieres expresar con eso? }\end{array}$ & $\begin{array}{l}\text { El objetivo de esta pregunta es determinar si el profesor } \\
\text { es consciente del uso de metáforas comentadas anterior- } \\
\text { mente en su discurso. }\end{array}$ \\
\hline $\begin{array}{l}P \text {. Hombre! - supongo que es absolutamente inconsciente, porque eso yo no lo pien- } \\
\text { so, yo no digo; me voy a mover de esta manera o de la otra, ............qué hacen? ¡, yo } \\
\text { creo que son gestos para reforzar la idea que expresas,... para reforzarla--- no! Es de- } \\
\text { cir; viene por aquí, pues, debería señalar por donde pasaría la función,......siguiendo,.. } \\
\text { eh!.. imaginando que tu sigues la función dando valores a la } x \text { de manera creciente, } \\
\text { pues, cual sería la imagen--- o --- el--- trazado que seguiría la curva no! ---- me parece- } \\
\text {---son cosas que---- eh! --- claro no me las he planteado, sí que gesticulas pensando } \\
\text { que refuerzas lo que tú estás diciendo ¿no?--- esto está,...----esto está claro. Porque, si } \\
\text { no, sería también muy aburrido dar un discurso ---- sin......... no, no llegará de la misma } \\
\text { manera, esto está claro. }\end{array}$ & $\begin{array}{l}\text { Deja en evidencia que lo hace de manera totalmente } \\
\text { inconsciente. } \\
\text { Asume inmediatamente que los gestos refuerzan una idea. } \\
\text { El profesor en su clase ha explicado algo estático en tér- } \\
\text { minos dinámicos, lo que supone una metáfora que no tiene } \\
\text { controlada, y no es consciente de sus consecuencias. }\end{array}$ \\
\hline \multicolumn{2}{|l|}{ E. ................ no, no estático tampoco, pues, sería ........ } \\
\hline \multicolumn{2}{|l|}{ P. - ----------- Tratar de reforzar, tratar de reforzar la idea,...--- claro. } \\
\hline $\begin{array}{l}E \text {. Mueves las manos cuando explicas algunas partes de la gráfica y cuando } \\
\text { dices: «la } x \text { crece», «el numerador crece más que el denominador». ¿Siem- } \\
\text { pre explicas las gráficas de esta manera, o sea, siempre has explicado las } \\
\text { gráficas moviendo las manos y con esta clase de expresiones: «aquí viene } \\
\text { aquí», «aquí pasa por aquí»... }\end{array}$ & $\begin{array}{l}\text { El objetivo es saber si es habitual en él este tipo de ac- } \\
\text { ciones. }\end{array}$ \\
\hline P. Sí & $\begin{array}{l}\text { Inmediatamente reconoce que el uso de gestos y el uso } \\
\text { de este tipo de metáforas forman parte habitual de su } \\
\text { práctica profesional. }\end{array}$ \\
\hline $\begin{array}{l}E . \text {... ¿Podría uno explicar el gráfico estático sin movimiento? ¿Crees que es } \\
\text { mejor para ellos darle un poco de movimiento? }\end{array}$ & $\begin{array}{l}\text { El objetivo de esta pregunta es saber qué motivos son los } \\
\text { que le llevan a explicar las gráficas en términos dinámi- } \\
\text { cos, en lugar de hacerlo de manera estática. }\end{array}$ \\
\hline $\begin{array}{l}\text { P. ----- Sí, sí, por supuesto. Claro, claro, yo creo que,... hay un eslogan en la moda } \\
\text { que dice «la arruga es bella» es un eslogan publicitario para la moda «pues yo } \\
\text { creo,... yo decía entonces ¡el dinamismo es bello!...atrae.....es bello, en clase no es } \\
\text { lo mismo un discurso quieto y tal, y decir las cosas muy bien dichas que ver algo } \\
\text { que se mueva, al alumno si hay algo que se mueva, eso lo mira más, entonces en } \\
\text { cualquier sección intento.........no puedes abusar,.... pero si hay algún momento } \\
\text { que tu traes alguna cosa que se mueve, eso centra más la atención, por ejemplo } \\
\text { en geometría,... pues, yo traía algunos chavales a propuesta de aquel libro rojo de } \\
\text { la Emma Castelnuovo, de las transformaciones de las figuras, de cualquier figura, } \\
\text { de los cuadriláteros,... bueno entonces eso se construye con unas varillas, se pue- } \\
\text { den ir moviendo, hace años yo lo construí, pues con esta idea,......el dinamismo } \\
\text { atrae,... y entonces hay que introducir elementos de éstos, de que el alumno vea } \\
\text { que hay cosas que se mueven, el alumno,.....ja ver qué pasa! Por lo menos por } \\
\text { unos momentos se interesa a ver qué pasa. }\end{array}$ & $\begin{array}{l}\text { Corrobora lo que ya ha dicho antes: su objetivo es «faci- } \\
\text { litar la comprensión del concepto» y trata de justificar su } \\
\text { uso con argumentos relativamente triviales. No obstante, } \\
\text { entre estos argumentos destaca la importancia que da este } \\
\text { profesor al aspecto dinámico como elemento que puede } \\
\text { facilitar la atención de los alumnos. }\end{array}$ \\
\hline $\begin{array}{l}E . ~ ¿ Y \text { no has pensado que de pronto eso les traiga algún error conceptual, } \\
\text { de interpretación? }\end{array}$ & El objetivo es cuestionar su discurso. \\
\hline $\begin{array}{l}\text { P.---No, i que provoque..... el hecho de que, }, . . . . . \text { algún error concep- } \\
\text { tual. Que provoque algún error conceptual, como, como cuál, por ejemplo? }\end{array}$ & $\begin{array}{l}\text { Él no tiene idea de qué clase de errores le estoy hablando, } \\
\text { lo cual pone de manifiesto que este profesor no es cons- } \\
\text { ciente de que el uso de metáforas, además de facilitar la } \\
\text { comprensión, también puede dificultarla. }\end{array}$ \\
\hline$E$. Por ejemplo, que un punto no se mueve, que un punto es.......... & $\begin{array}{l}\text { El objetivo es poner un ejemplo de las dificultades que pue- } \\
\text { den aparecer por la interpretación literal de la metáfora. }\end{array}$ \\
\hline $\begin{array}{l}\text { P.--- No el punto no se mueve, la función tampoco, pero.....lo ves...cuando di- } \\
\text { ces que pasa, no es como un tren que pasa, sino que el trazado pisa esos puntos, } \\
\text { yo no lo he pensado, no creo que esto.....no he detectado que las confusiones } \\
\text { vengan por aquí, no lo he pensado, no creo, no, no... }\end{array}$ & $\begin{array}{l}\text { Si bien se cuestiona por un momento su discurso, en el } \\
\text { fondo sigue considerando que el uso de las metáforas } \\
\text { facilita la comprensión y no da importancia a las posibles } \\
\text { dificultades que pueda generar. }\end{array}$ \\
\hline
\end{tabular}


De esta entrevista podemos observar, entre otros, los siguientes hechos significativos:

1) Las respuestas del profesor corroboran nuestra hipótesis sobre el uso de las metáforas en su discurso. El profesor no es consciente de que las usa y, por tanto, no las controla, de manera que puede ocurrir que algunos alumnos conceptualizen situaciones de una manera incorrecta. Mediante la metáfora estructuramos una situación en términos de otra, y se corre el peligro de trasladar aspectos del dominio de partida que no son aplicables en el dominio de llegada. Por ejemplo, suponer que «una gráfica es la traza de un punto que se mueve como un objeto» puede facilitar que algunos alumnos entiendan que la gráfica es como un coche, siempre el mismo, que se mueve sobre una carretera, en lugar de considerar la gráfica como una carretera formada por un conjunto de puntos diferentes.

2) Como consecuencia de las preguntas del entrevistador, el profesor se da cuenta de que usa metáforas, pero considera que su uso facilita la comprensión y no da importancia a las posibles dificultades que pueda generar en sus alumnos. De hecho, considera que el uso de metáforas no genera ningún tipo de error conceptual en sus alumnos.

Nuestro siguiente objetivo fue determinar el efecto que producen las metáforas utilizadas por el profesor en la comprensión de sus alumnos. A tal efecto, confeccionamos el siguiente cuestionario para que fuese contestado por 9 alumnos escogidos al azar entre todos los que participaron en la clase grabada en vídeo.

\section{Descripción del cuestionario}

Se trata de un cuestionario formado por 6 preguntas semiabiertas cuyo objetivo era determinar el efecto que producen, en la comprensión de los alumnos, las metáforas utilizadas por el profesor al explicar los siguientes conceptos: crecimiento, decrecimiento y gráfica de una función

\section{Describe la siguiente gráfica}

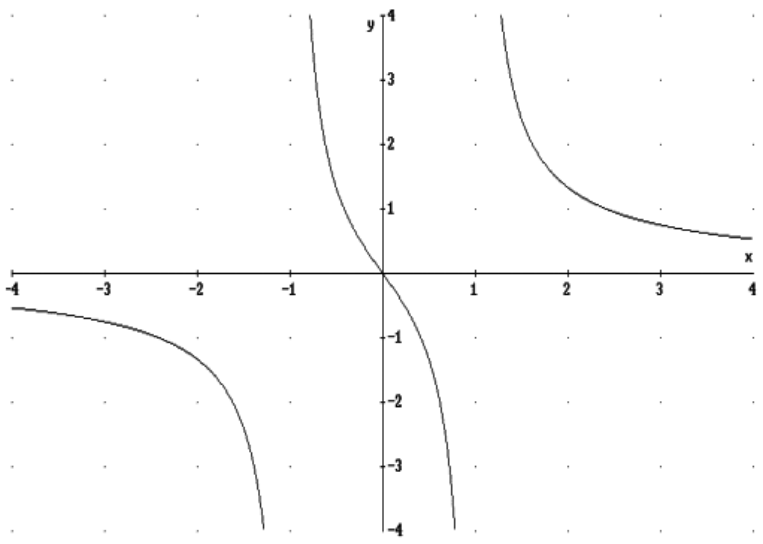

En esta primera pregunta se da libertad al alumno para que describa con sus propias palabras una gráfica. El objetivo de la pregunta es determinar si utiliza metáforas dinámicas y, más en concreto, términos que el profesor utilizó en clase, tales como: pasa por..., viene de..., se va hasta..., etc.

\section{La gráfica, ¿crece o decrece? Explica por qué}

El objetivo de la pregunta es determinar de qué metáforas se vale para explicar los conceptos de crecimiento y decrecimiento y si lo hace con términos análogos a los que el profesor utilizó en clase, tales como: sube, baja, viene del infinito, va al infinito, etc.

\section{¿Qué les pasa a los puntos de la curva en las asíntotas verticales?}

Esta pregunta, formulada en términos parecidos a los utilizados por el profesor en su explicación, tiene por objetivo determinar si el alumno responde usando implícitamente el lenguaje de las sucesiones, con respuestas como «cuando los valores de la abscisa se aproximan a 1 por la derecha, los valores de la ordenada son tan grandes como se quiera» o bien si lo hace en un lenguaje menos preciso con frases como «me puedo aproximar tanto como quiera». Se trata de establecer hasta qué punto hay coherencia entre las respuestas del alumno y la explicación del profesor. En el primer caso el alumno ha de considerar diferentes valores para la abscisa y para la ordenada, mientras que, en el segundo, el alumno considera un solo valor de la abscisa o de la ordenada, el cual «se aproxima», «se hace grande», etc.

¿Crees que hay alguna diferencia entre los puntos de abscisa $x=a \quad y \quad x=b$ ?

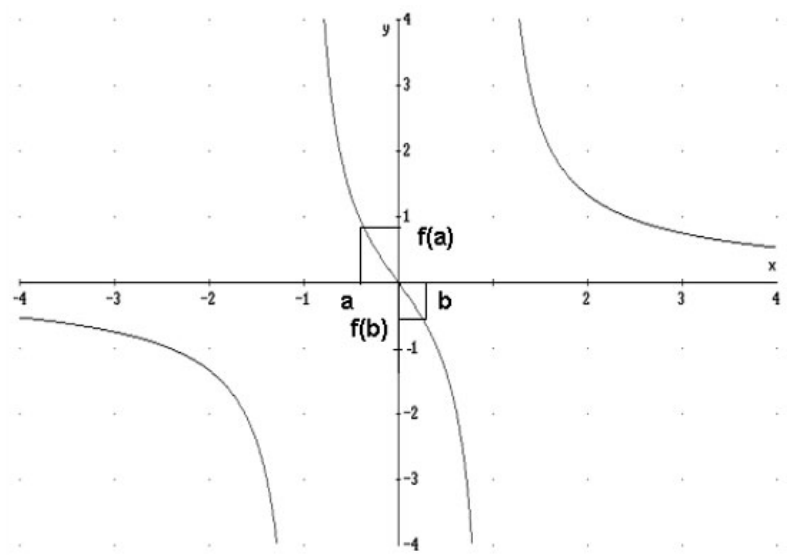

Con esta pregunta se pretendía observar qué tipo de argumentación utilizaba el alumno para describir la diferencia entre los dos puntos. Se trataba de ver si los alumnos argumentaban: $a$ ) con los nuevos contenidos introducidos por el profesor -crecimiento o decrecimiento en un punto, concavidad y convexidad en un punto-; 
b) con metáforas orientacionales, como por ejemplo «la imagen de $a$ está arriba y la de $b$ abajo»; $c$ ) con el signo de las coordenadas, como, por ejemplo, «la abscisa y la ordenada de un punto son positivas, mientras que las del otro son negativas»; $d$ ) con los dos últimos tipos de argumentaciones, como, por ejemplo: «es positivo porque está a la derecha o arriba», «es negativo porque está a la izquierda o abajo», etc.

\section{¿Conoces algún fenómeno real que se pueda asociar a la gráfica?}

El objetivo era saber si el alumno era capaz de adaptar la gráfica a una situación cotidiana.

\section{Conoces varias formas de saber cuando una gráfica crece o decrece. ¿Cuáles? Explica tu repuesta}

En esta pregunta no se especifica si se trata de crecimiento o decrecimiento en un punto o bien en un intervalo. El objetivo es determinar si los alumnos responden utilizando de manera implícita la metáfora «la gráfica de una función es la traza que deja un punto que se mueve sobre la gráfica», con frases como «viene de arriba abajo», etc., o bien utilizando el signo de la derivada.

\section{Respuestas al cuestionario}

Las respuestas de los alumnos fueron las siguientes:

\begin{tabular}{|c|c|c|c|c|c|c|}
\hline Alumnos & Describe la gráfica & $\begin{array}{c}\text { La gráfica, } \\
\text { ¿crece } \\
\text { o decrece? } \\
\text { Explica por qué }\end{array}$ & $\begin{array}{l}\text { ¿Qué les pasa } \\
\text { a los puntos de } \\
\text { la gráfica en } \\
\text { las asíntotas } \\
\text { verticales? }\end{array}$ & $\begin{array}{c}\text { ¿Crees que } \\
\text { hay alguna } \\
\text { diferencia entre } \\
\text { los puntos de } \\
\text { abscisa } x=a \\
\text { y } x=b ?\end{array}$ & $\begin{array}{l}\text { ¿Conoces } \\
\text { algún } \\
\text { fenómeno } \\
\text { real que } \\
\text { se pueda } \\
\text { asociar a } \\
\text { la gráfica? }\end{array}$ & $\begin{array}{l}\text { Conoces varias formas } \\
\text { de saber cuando una } \\
\text { gráfica crece o decrece. } \\
\text { ¿Cuáles? Explica tu } \\
\text { respuesta. }\end{array}$ \\
\hline 1 & $\begin{array}{l}\text { Dominio }\left\{\begin{array}{l}\{1 \\
1\end{array}\right. \\
\text { Tiene asíntotas } \\
\text { horizontales y } \\
\text { verticales } \\
\text { De }-\infty \text { a }-1 \text { es convexa } \\
\text { De } 1 \text { a } 0 \text { cóncava } \\
\text { De } 0 \text { a } 1 \text { convexa } \\
\text { De } 1 \text { a }+\infty \text { cóncava }\end{array}$ & $\begin{array}{l}\text { Decrece } \\
\text { De }-\infty \text { a }-1 \\
\text { decrece, de } 1 \text { a } \\
+\infty \text { decrece, de } \\
-1 \text { a } 1 \text { decrece }\end{array}$ & $\begin{array}{l}\text { Que van paralelas a } \\
\text { ellas hasta infinito } \\
\text { y nunca la llegan } \\
\text { a tocar }\end{array}$ & $\begin{array}{l}\text { Que } a \text { es negativa } \\
\text { y } b \text { es positiva }\end{array}$ & No lo sé & \\
\hline 2 & $\begin{array}{l}\text { Asíntotas verticales } \\
(-1,0)(1,0) \\
\text { Asíntotas horizontales } \\
(0,0) \\
\text { Intervalo de } \\
\text { decrecimiento } \\
(-\infty,-1)(-1,1)(1,+\infty)\end{array}$ & $\begin{array}{l}\text { Decrece, porque } \\
\text { va de más } \\
\text { infinito }(+\infty) \text { a } \\
\text { menos infinito } \\
(-\infty) \text { desde el } \\
\text { punto de vista } \\
\text { del eje de las } y\end{array}$ & $\begin{array}{l}\text { Que tienden hacia } \\
\text { la asíntota pero } \\
\text { nunca la tocan, van } \\
\text { hasta infinito }(+\infty \\
\text { a la derecha y }-\infty \\
\text { por la izquierda) }\end{array}$ & $\begin{array}{l}\text { Son de signos } \\
\text { opuestos }\end{array}$ & No & $\begin{array}{l}\text { Sí, mirando si va de } \\
\text { izquierda a derecha hacia } \\
\text { los números negativos } \rightarrow \\
\text { y si va de izquierda } \\
\text { a derecha hacia los } \\
\text { números positivos (de la } \\
\text { U) la función crece }\end{array}$ \\
\hline 3 & $\begin{array}{l}\text { Las asíntotas verticales } \\
\text { están en } x=-1 \text { y } x=+1 \\
\text { Intervalos de } \\
\text { crecimiento }(-\infty,-1) \\
(-1,1)(+1,+\infty) \text {. Las } \\
\text { asíntotas horizontales } \\
\text { también tienden hacia } \\
0 \text { cuando más se alejan } \\
\text { de } x=0\end{array}$ & $\begin{array}{l}\text { De }-\infty \text { hasta } \\
-1 \text { decrece; de } \\
-1 \text { a }+1 \text { también } \\
\text { decrece; y de }+1 \\
a+\infty \text { decrece }\end{array}$ & $\begin{array}{l}\text { Que se acercan a } \\
-1 \text { o a }+1\end{array}$ & $\begin{array}{l}x=b \text { está en el lado } \\
\text { positivo y } x=a \text { está } \\
\text { en el lado negativo }\end{array}$ & & \\
\hline 4 & $\begin{array}{l}\text { Decrece } \\
\text { La gráfica tiene dos } \\
\text { asíntotas verticales, una } \\
\text { pasa por el punto }-1 \text {, } \\
\text { la otra por el punto } 1 \text {, } \\
\text { una asíntota horizontal } \\
\text { es } 0 \text {, no tiene punto de } \\
\text { inflexión, la gráfica es } \\
\text { una función racional }\end{array}$ & $\begin{array}{l}\text { La gráfica } \\
\text { decrece }\end{array}$ & $\begin{array}{l}\text { Que la curva se va } \\
\text { hacia infinito }\end{array}$ & $\begin{array}{l}\text { Sí, que el punto } \\
x=a \text { es }+y \text { el } \\
\text { punto } x=b \text { es - }\end{array}$ & & $\begin{array}{l}\text { Observando } \\
\text { Decrece } \\
\text { la curva está } \\
\text { inclinada hacia } \\
\text { abajo } \\
\text { o bien tiende hacia } \\
\text { abajo de esta otra } \\
\text { forma } \\
\text { Crece } \\
\text { desde abajo la } \\
\text { la curva se inclina hacia } \\
+\infty\end{array}$ \\
\hline
\end{tabular}




\begin{tabular}{|c|c|c|c|c|c|c|}
\hline 5 & $\begin{array}{l}\text { Hay dos asíntotas para } \\
x=-1 ; x=1 \text { verticales } \\
\text { La gráfica corresponde } \\
\text { a una función racional }\end{array}$ & $\begin{array}{l}\text { Tiene intervalos } \\
\text { de crecimiento } \\
\text { y decrecimiento }\end{array}$ & $\begin{array}{l}\text { Que las funciones } \\
\text { tienen valores muy } \\
\text { grandes o muy pe- } \\
\text { queños }\end{array}$ & & No & $\begin{array}{l}\text { Sí: 1) por el signo de la } \\
\text { primera derivada } f(x) \\
\text { 2) por la concavidad o } \\
\text { convexidad de la gráfica }\end{array}$ \\
\hline 6 & $\begin{array}{l}\text { En esta gráfica hay dos } \\
\text { asíntotas verticales en } \\
1 \text { y }-1 \text {, eso quiere decir } \\
\text { que la función existe } \\
\text { por todos los } \mathrm{R} \text { menos } \\
1 \text { y }-1 \text {. Para estos } \\
\text { últimos valores men- } \\
\text { tados últimamente la } \\
\text { función no existe. Un } \\
\text { punto de corte en }(0,0) \\
\text { de inflexión (la función } \\
\text { pasa de cóncava a con- } \\
\text { vexa) de }(-\infty,-1) \text { fun- } \\
\text { ción decreciente }(-1 \\
\text { a } 0) \text { decreciente }(0,1) \\
\text { decreciente }(1,+\infty) \text { de- } \\
\text { creciente }\end{array}$ & Decrece & $\begin{array}{l}\text { Que nunca la curva } \\
\text { llegará a tocar las } \\
\text { asíntotas }\end{array}$ & $\begin{array}{l}\text { Que la } a \text { es ne- } \\
\text { gativa y la } b \text { es } \\
\text { positiva }\end{array}$ & No & $\begin{array}{l}\text { Una mirando el gráfico. } \\
f(x)>0 \rightarrow \text { función cre- } \\
\text { ciente } \\
f(x)<0 \rightarrow \text { función de- } \\
\text { creciente }\end{array}$ \\
\hline 7 & $\begin{array}{l}\text { Esta gráfica pertenece } \\
\text { a una función racional } \\
\text { porque tiene disconti- } \\
\text { nuidades (asíntotas) que } \\
\text { hace que la gráfica esté } \\
\text { dividida en diferentes } \\
\text { partes }\end{array}$ & $\begin{array}{l}\text { Tiene diferentes } \\
\text { partes: } \\
\text { 1a) decrece } \\
\text { 2a) decrece } \\
\text { 3a) decrece } \\
\text { 4a) decrece }\end{array}$ & $\begin{array}{l}\text { Que sufren una dis- } \\
\text { continuidad y que } \\
\text { no existen }\end{array}$ & $\begin{array}{l}\text { Sí, que } x=a \text { es } \\
\text { negativa y } x=b \\
\text { es positivo }\end{array}$ & & $\begin{array}{l}\text { 1) Observando el gráfico } \\
\text { 2) Dando valores a } x \text { y ob- } \\
\text { servar si crece o decrece }\end{array}$ \\
\hline 8 & & Decrece & & $\begin{array}{l}\text { La } a \text { es negativa y } \\
\text { la } b \text { es positiva }\end{array}$ & No & \\
\hline 9 & $\begin{array}{l}\text { Int: }(-\infty,-1) \text { la fun- } \\
\text { ción decrece porque } \\
\text { cuando la función se } \\
\text { acerca a la asíntota } \\
\text { tie-de al }-\infty \text { y, por lo } \\
\text { tanto, decrece. } \\
\text { Int: }(-1,1) \text { en } x=-1 \\
\text { hay una asíntota y en } \\
x=1 \text { otra, en este in- } \\
\text { tervalo la función va de } \\
+\infty \text { a }-\infty \text {, por lo tanto, } \\
\text { también decrece. } \\
\text { Int: }(1,+\infty) \text { la función } \\
\text { vuelve a decrecer, ésta } \\
\text { viene de la asíntota en } \\
+\infty \text { y decrece, se va } \\
\text { al }+\infty \text { por la asíntota } \\
\text { horizontal en } y=0\end{array}$ & $\begin{array}{l}\text { Decrece } \\
\text { Se distingue cla- } \\
\text { ramente }\end{array}$ & $\begin{array}{l}\text { No existe nunca ni } \\
\text { P. I. En las asíntotas } \\
\text { verticales }\end{array}$ & $\begin{array}{l}\text { Sí, en los dos puntos } \\
\text { la } f(x) \text { decrece, pero, } \\
\text { cuando } x=a \text { la } \mathrm{f}(x) \\
\text { es cóncava y, cuan- } \\
\text { do } x=b, \text { la función } \\
\text { es convexa }\end{array}$ & $R-\{-1,1\}$ & $\begin{array}{l}\text { Sí, por los intervalos de } \\
\text { crecimiento o decreci- } \\
\text { miento o por los inter- } \\
\text { valos de concavidad y } \\
\text { convexidad }\end{array}$ \\
\hline
\end{tabular}

Las respuestas de los alumnos permiten extraer las siguientes conclusiones:

Con relación a la pregunta 1 , se observa que 8 de los 9 alumnos dieron una descripción de la gráfica utilizando algunas de las características introducidas por el profesor (dominio, intervalos de decrecimiento, asíntotas, etc.), mientras que 1 (el núm. 8) respondió en blanco. También hay que destacar que 3 de ellos reconocieron la gráfica como perteneciente a la familia de las racionales. En las respuestas de los alumnos 3 y 9 se observa una clara influencia del discurso metafórico del profesor.
Con relación a la pregunta 2 , se observa que los alumnos se limitan a responder sin dar ninguna explicación de su respuesta. Los únicos que dan algún tipo de justificación son el número 9, que se limita a decir «Se distingue claramente» y el número 2, que da una explicación en términos metafóricos.

Del análisis conjunto de las respuestas 1 y 2 se observa que cuatro alumnos (2, 3, 4 y 9), en su descripción de la gráfica, utilizaron expresiones como: «pasa por», «va de», «tiende hacia infinito», «cuando más se alejan de», etc. De esta manera, nuestra hipótesis de que un sector 
importante de alumnos utilizaría un tipo de vocabulario metafórico parecido al del profesor en su explicación se corroboró.

Con relación a las repuestas a la pregunta número 3 , predominaron las respuestas en términos metafóricos como: «la curva se va hacia infinito», «que nunca la curva llegará a tocar las asíntotas», etc. Ninguno de ellos respondió, por ejemplo, que la recta $x=1$ era una asíntota vertical porque el límite de la función cuando $x \rightarrow 1^{+}$era $+\infty$ y cuando $x \rightarrow 1^{-}$era $-\infty$, de acuerdo con las definiciones que el profesor había dado previamente y que ellos tenían recogidas en sus cuadernos.

Con relación a las repuestas de la pregunta número 4, predominaron las que sólo daban argumentos basados en el signo de las coordenadas (siete de los nueve alumnos). Uno no respondió y sólo uno asoció esta diferencia al hecho de que pertenecen a trozos diferentes de la curva: uno a la parte cóncava y el otro a la convexa.

Con relación a la pregunta 5, todos los alumnos, tal como esperábamos, contestaron que no conocían ningún fenómeno cotidiano asociado a esta representación gráfica. La causa hay que buscarla en la propia dificultad de la pregunta, pero también resulta significativo que no haya ninguna respuesta, ni siquiera incorrecta, porque pone de manifiesto la inexperiencia de estos alumnos para responderla, a causa de que el proceso de estudio que han seguido no incluye ningún problema contextualizado. Solamente han trabajado en clase la siguiente situación: dada la fórmula de una función, representarla gráficamente.

Con relación a la pregunta 6 , tres alumnos no respondieron, y seis de los nueve reconocen el crecimiento y decrecimiento de una curva por el mero hecho de observarla visualmente. Dos de ellos apoyan su afirmación visual con expresiones metafóricas del tipo «la curva está inclinada hacia abajo» y sólo dos responden utilizando el signo de la derivada. Hay que destacar que ninguno utilizó claramente la definición de crecimiento en un punto o en un intervalo dada por el profesor. Por ejemplo, no hubo ninguna respuesta del tipo: si $a<b$, entonces $f(a)<f(b)$. Sólo en la respuesta del alumno 7 se puede intuir este tipo de argumentación.

Como conclusión general podemos decir que las respuestas al cuestionario confirman nuestra suposición de que el uso de metáforas en el discurso del profesor produce efectos significativos en la comprensión de los alumnos. El objetivo que se había marcado el profesor era la enseñanza de una técnica analítica de representación gráfica de funciones. Para facilitar su comprensión utiliza de manera inconsciente, y según él de manera inocua, expresiones que implican metáforas del tipo «la gráfica es la traza que deja un punto que se mueve sobre la gráfica» o similares. Las respuestas de los alumnos sugieren claramente que este tipo de metáforas tiene mucho más peso del que normalmente se considera, ya que son las que muchos de ellos utilizan para responder a las preguntas, en lugar de las definiciones más precisas que tienen recogidas en sus cuadernos.

\section{CONCLUSIONES FINALES}

1) En este trabajo hemos puesto de manifiesto que, en la enseñanza de la representación gráfica de funciones en el bachillerato, se produce, con una cierta generalidad, el siguiente fenómeno: el profesor usa de manera poco consciente expresiones que sugieren, entre otras, metáforas del tipo «la gráfica de una función se puede considerar como la traza que deja un punto que se mueve sujeto a determinadas condiciones» $-\mathrm{o}$ bien una variación de esta metáfora: «La gráfica de una función se puede considerar como la traza que deja un punto que se mueve sobre la gráfica». Estas metáforas no son inocuas y producen efectos significativos en la comprensión de los alumnos.

2) Las causas que puede explicar este fenómeno son complejas. Por una parte, tal como señalan Lakoff y Núñez, el uso de la metáfora es fundamental en la comprensión de cualquier tema $-\mathrm{y}$, por tanto, en su explicación-; ahora bien, en nuestra opinión, también puede haber causas relacionadas con las matemáticas, ya que la representación gráfica de funciones necesita, además de una descripción en términos globales, la introducción de conceptos locales tales como crecimiento y decrecimiento en un punto, etc. Estos conceptos locales presentan una gran dificultad para los alumnos, motivo por el cual, a nuestro entender, los profesores los dejan en un segundo plano y prefieren utilizar explicaciones dinámicas en las que el uso de la metáfora es fundamental.

3) El uso de determinados graficadores informáticos facilita la recuperación, aunque sea inconsciente, del uso de metáforas dinámicas. Actualmente hay graficadores informáticos y calculadoras gráficas cuyo uso conlleva entender la gráfica como la trayectoria de un punto, lo cual implica estructurar el concepto de gráfica en términos cinéticos y se refleja en el lenguaje utilizado (punto que se mueve). Además de los programas informáticos, los gestos del profesor conllevan, aunque sea de manera inconsciente, el uso de metáforas dinámicas.

4) El uso de metáforas dinámicas tiene sus ventajas y sus inconvenientes. En nuestra opinión, no se trata de renunciar a ellas, sino que el objetivo ha de ser un uso controlado, siendo conscientes de que no todo son ventajas. 


\section{NOTAS}

${ }^{1}$ Las referencias a los alumnos están contextualizadas en el bachillerato de la comunidad autónoma de Catalunya (España). Son alumnos de 16-17 años.

${ }^{2}$ Con relación a esta actividad, no nos interesa entrar a valorar si está bien formulada o no, o si se puede substituir por otra más adecuada. Sólo nos hemos limitado a seleccionar una actividad clásica de un libro de texto -Grup ZERO (1981). Les funcions lineals i quadràtiques. Barcelona: Vicens Vives (p. 20)-, que con algunas modificaciones se puede encontrar en algunos libros actuales de ESO, y a la cual hemos añadido los dos últimos apartados.
${ }^{3}$ En Font y Peraire (2001) y Font (2001) se puede encontrar un desarrollo amplio de estas cuatro metáforas.

${ }^{4}$ Se puede consultar un resumen de esta investigación en Enseñanza de las Ciencias, 18 (3) pp. 497-498.

${ }^{5}$ En la trascripción hemos utilizado la siguiente nomenclatura:

...... Significa que hubo una pausa de tiempo pero se completó la idea con gestos físicos.

------ Pausa de tiempo, proporcional a la longitud.

\section{REFERENCIAS BIBLIOGRÁFICAS}

ENGLISH, L.D. (ed.) (1997). Mathematical reasoning: Analogies, metaphors and images. Mahwah, N.J.: Erlbaum.

FONT, V. (2000). Procediments per obtenir expressions simbòliques a partir de gràfiques. Aplicacions a les derivades. Tesis doctoral no publicada. Universitat de Barcelona.

FONT, V. (2001). Expresiones simbólicas a partir de gráficas. El caso de la parábola. Revista EMA, 6(2), pp. 180-200.

FONT, V. y PERAIRE, R. (2001). Objetos, prácticas y ostensivos asociados. El caso de la cisoide. Educación Matemáti$c a, 13(2)$, pp. 55-67.

LAKOFF, G. y JOHNSON, M. (1991). Metáforas de la vida cotidiana. Madrid: Cátedra.

LAKOFF, G. y NÚÑEZ, R. (1998). Conceptual metaphor in mathematics, en Koenig J.P. (ed.). Discourse and Cognition: Bridging the Gap, pp. 219-237. Stanford: CSLI-Cambridge.

LAKOFF, G. y NÚÑEZ, R. (2000). Where mathematics comes from: How the embodied mind brings mathematics into being. Nueva York: Basic Books.
NÚÑEZ, R. (2000). Mathematical idea analysis: What embodied cognitive science can say about the human nature of mathematics, en Nakaora T. y Koyama M. (eds.). Proceedings of the 24th Conference of the International Group for the Psychology of Mathematics Education (vol. 1, pp. 3-22). Hiroshima: Hiroshima University.

NÚÑEZ, R. y LAKOFF, G. (1998). What did Weierstrass really define? The cognitive structure of natural and $\xi-\partial$ continuity. Mathematical Cognition, 4(2), pp. 85-101.

PIAGET, J. (1979). Los problemas principales de la epistemología de la matemática, en J. Piaget (comp.). Epistemología de la matemática, pp. 147-182. Buenos Aires: Paidós.

PIMM, D. (1990). El lenguaje matemático en el aula. Madrid: MEC-Morata.

VAN DORMOLEN, J. (1991). Metaphors Mediating the Teaching and Understanding of Mathematics, en Bishop J. y Melling Olsen S. (eds.). Mathematical Knowledge: Its Growth Through Teaching, pp. 89-106. Dordrecht: Kluwer A.P. 